\title{
On the Computation of Mass Fluxes for Eulerian Transport Models from Spectral Meteorological Fields
}

\author{
Arjo Segers ${ }^{1}$, Peter van Velthoven ${ }^{1}$, Bram Bregman $^{2}$, and Maarten $\mathrm{Krol}^{2}$ \\ 1 Royal Netherlands Meteorological Institute (KNMI), \\ P.O. Box 201, 3730 AE, De Bilt, The Netherlands \\ $\{$ Arjo.Segers, Velthove $@$ @knmi.nl \\ 2 Institute for Marine and Atmospheric Research (IMAU), \\ University of Utrecht, The Netherlands \\ $\{$ A.Bregman, M.Krol\}@phys.uu.nl
}

\begin{abstract}
A procedure is introduced to compute mass fluxes and mass tendencies for a Eulerian transport model from spectral fields in a consistent way. While meteorological forecast models are formulated in terms of vorticity, divergence, and surface pressure expanded in spherical harmonics, Eulerian transport models, widely used for tracer and climate studies, require wind fields or mass fluxes on a regular grid. Here horizontal and vertical mass fluxes are computed directly from the spectral data to stay as close to the representation in the meteorological model as possible. The presented procedure can be applied to compute fluxes for global as well as limited area models.
\end{abstract}

\section{Introduction}

The study of climate and atmospheric composition with three dimensional global models requires accurate simulation of tracer transport. Horizontal winds increasing in magnitude from the surface upwards are the driving force for global transport and mixing of trace gases. The exchange of trace gases between the different atmospheric compartments such as the mid latitude troposphere and stratosphere is a key process for the impact of human activity on climate. For accurate simulation of transport, three dimensional chemistry-transport models (CTMs) are often equipped with sophisticated and expensive advection routines [1]. However, less attention is paid to the numerical procedure to compute mass fluxes required for the advection and to interpolate them to the model grid. A model comparison by [2] showed that performance of CTMs strongly depends on how the meteorological model input is used. Comparison of simulated ozone concentrations with aircraft measurements showed significant differences between chemistry transport models, in spite of a common source of meteorological data (ECMWF) and usage of similar advection and identical chemistry schemes.

The mass fluxes used as input for tracer models are derived from wind fields from a meteorological model. Most tracer models use analyzed or forecasted wind 
fields from a weather forecast or climate model. Data from meteorological forecast models have the advantage of being based on assimilation of meteorological measurements. A disadvantage is that the data is only available at discrete time instants 3 or 6 hours apart. Nowadays most operational meteorological forecast models are formulated in terms of vorticity and divergence rather than wind components. Most models represent these fields in terms of spherical harmonics. Tracer transport models however expect input of mass fluxes through boundaries of grid boxes or wind fields at the grid cell centers. Therefore, a method should be developed to compute mass fluxes in a consistent and accurate way from the spectral fields.

A method often applied is interpolation of the spectral wind fields to a high resolution regular grid. Integration of these wind fields over the boundaries of grid boxes then provides the required mass fluxes. If the high resolution grid does not match with the grid of the tracer model, additional interpolations are required. Vertical fluxes are then computed from horizontal divergence and boundary conditions of zero flux through the top and bottom. This method has for instance been used in older versions of CTM2 [3] and TM3 [2]. Although the horizontal fluxes computed in this way are quite accurate, the errors in the vertical fluxes can be quite large. The vertical velocities are very small (a few $\mathrm{cm}$ per second) in comparison with the horizontal winds, which are of the order of several to tens of meters per second; this is a consequence of near geostrophic balance [4]. If interpolated wind fields are used to compute the vertical ones, small interpolation errors easily lead to large errors in the vertical wind [4]. In [2] it is stated that for this reason the use of interpolated fields should be avoided.

To avoid unnecessary interpolations, the mass fluxes should be computed from spectral fields directly. The input for the TOMCAT model is computed from vorticity and divergence using integrated spherical harmonic functions [5]. In particular, the vertical fluxes are computed from divergence fields to stay as close to the parent meteorological model as possible. Near the surface, where the surface pressure acts on the hybrid vertical coordinate, the mass fluxes computed for TOMCAT are corrected to preserve the tracer mixing ratio.

This paper proposes a consistent scheme for computation of mass fluxes from spectral fields of vorticity, divergence, and surface pressure, avoiding the use of interpolated wind fields. The next section describes the horizontal and vertical grid of a general Eulerian model, and requirements for the mass fluxes. A short discussion of the integration of spectral fields is given in 3 . The algorithm proposed in section 4 computes a consistent set of three dimensional fluxes, in balance with the surface pressure tendency. The method as described here is suitable for computation of mass fluxes for a global tracer transport model with rectangular grid boxes in the lat/lon plane. All equations assume a vertical coordinate system with hybrid $\sigma /$ pressure levels, for which pressure and $\sigma$ levels are special cases. Modifications needed for non-regular discrete grids or other vertical coordinate systems are straightforward. The new system of mass fluxes has been evaluated with the TM3 model; a comparison between old and new formulation is provided in section 5 . 


\section{Definition of the tracer model}

We consider a general Eulerian transport model, defined on a three dimensional grid of hybrid $\sigma /$ pressure levels. For the vertical levels it is common practice to copy the set of vertical levels used by the meteorological model or a subset of it. In a system of hybrid $\sigma$ /pressure coordinates such as used by ECMWF [6], the vertical coordinate is a dimensionless parameter $\eta \in[0,1]$. All that is required for $\eta$ is that it is a function $h\left(p, p_{s}\right)$ of the pressure and surface pressure at a certain location, with the boundary $h\left(0, p_{s}\right)=0$ at the top and $h\left(p_{s}, p_{s}\right)=1$ at the surface. In the vertical there are $n_{\text {lev }}$ layers with boundaries or half levels at $\eta_{k-1 / 2}$ and $\eta_{k+1 / 2}$ and mid levels $\eta_{k}$. At the half levels, the pressure is specified by hybrid coefficients $a_{k+1 / 2}$ and $b_{k+1 / 2}$ :

$$
p\left(\lambda, \beta, \eta_{k+1 / 2}\right)=a_{k+1 / 2}+p_{s}(\lambda, \beta) b_{k+1 / 2} \quad[\mathrm{~Pa}]
$$

where $\lambda$ and $\beta$ denote the longitude and latitude respectively, both in radians. For integration of a field $F(\eta)$ between two half levels we will use the procedure used in the ECMWF model:

$$
\int_{\eta_{k-1 / 2}}^{\eta_{k+1 / 2}} F(\eta) \frac{\partial p}{\partial \eta} \mathrm{d} \eta \approx F_{k} \Delta p_{k}=F_{k}\left(\Delta a_{k}+p_{s} \Delta b_{k}\right)
$$

where $\Delta a_{k}=a_{k+1 / 2}-a_{k-1 / 2}$ and $\Delta b_{k}=b_{k+1 / 2}-b_{k-1 / 2}$. The horizontal grid will be supposed to consist of rectangular cells in the lat/lon plane. A cell $i$ then has a surface area of:

$$
A_{i}=\left[\lambda_{i}^{-}, \lambda_{i}^{+}\right] \times\left[\beta_{i}^{-}, \beta_{i}^{+}\right]=\Delta \lambda_{i} \times \Delta \beta_{i} \quad\left[\operatorname{rad}^{2}\right]
$$

The size and location of the cells is left undefined. With the algorithm introduced in section 4 we will be able to compute mass fluxes through all six boundaries of a box, independent of other boxes. In a simple configuration, the grid cells form a regular pattern with equal spacing in both the latitudinal and longitudinal direction. Irregular grids are however possible too: modern tracer models often use a regular or quasi-regular reduced Gaussian grid which implies irregular spacing in latitudinal direction and for each latitude band a different longitudinal spacing. The tracer model requires three entities which in theory completely define the airmass fluxes between the cells within a time interval $\left[t_{0}, t_{1}\right]$ :

1. the air mass distribution at $t_{0}$, for example in $\mathrm{kg}$ per grid box;

2. the horizontal mass fluxes in $\mathrm{kg} / \mathrm{s}$ through the boundaries of the grid cells, valid for the complete interval $\left[t_{0}, t_{1}\right]$;

3. the air mass distribution at $t_{1}$.

Knowledge of these entities implies that the vertical fluxes can be calculated from mass conservation. In here, we will however compute the vertical flux from the meteorological data too, in order to correct for errors in the horizontal fluxes. Mass conservation implies that the net flux into a box should equal the difference in mass between the beginning and end of the interval. The latter is given by the change in surface pressure in the case of time-independent vertical coefficients $a_{k}$ and $b_{k}$. 


\section{Spectral fields}

To compute the variables specified in the previous section, a meteorological model should provide wind fields and surface pressures. Meteorological models such as the ECMWF model solve the continuity equation on the sphere:

$$
\frac{\partial}{\partial t}\left(\frac{\partial p}{\partial \eta}\right)+\boldsymbol{\nabla} \cdot\left(\boldsymbol{v} \frac{\partial p}{\partial \eta}\right)+\frac{\partial}{\partial \eta}\left(\dot{\eta} \frac{\partial p}{\partial \eta}\right)=0
$$

where $\boldsymbol{v}$ is the horizontal wind and $\boldsymbol{\nabla}$ the horizontal gradient operator. The output of the model consists, amongst others, of fields of divergence $D=\boldsymbol{\nabla} \cdot \boldsymbol{v}$, the vertical component of the vorticity $\xi=(\boldsymbol{\nabla} \times \boldsymbol{v})_{\eta}$, and the natural logarithm of the surface pressure $\ln \left(p_{s}\right)$. These are available in the form of spectral coefficients. Their value at a certain point in grid space can be computed from the expansion:

$$
X(\lambda, \mu)=\sum_{m=-M}^{M} \sum_{n=|m|}^{N} X_{n}^{m} P_{n}^{m}(\mu) e^{i m \lambda}
$$

Here, $\mu=\sin (\beta)$, and $P_{n}^{m}$ denote the associated Legendre functions. For computation of mass distributions and fluxes, the spectral fields need to be integrated. An integral in the longitudinal direction can simply be solved analytically. In the latitudinal direction, integrals over the associated Legendre functions can be computed once to be used multiple times for expansion of the spectral sum using integrated rather than ordinary base functions. Since no simple analytic solutions exist for definite integrals of associated Legendre functions, the integrals over $P_{n}^{m}$ have to be solved numerically. This approach is for example used for preprocessing meteorological data in the TOMCAT model [5]. Apart from difficulties with oscillating Legendre functions at higher truncations, this method is not suitable for an integral over a product of spectral fields, a computation that is often needed in the hybrid level system when fields are multiplied with the surface pressure before integration (see for example equations (9-11) and (13-14) below). A better method is therefore to evaluate the spectral fields to a high resolution grid, and to use numerical quadrature afterwards.

\section{Computation of mass fluxes}

The proposed algorithm for computation of the mass fluxes and mass distributions for the tracer model consists of four stages.

\subsection{Computation of the mass distribution}

The mass distribution in $\mathrm{kg}$ per grid box at $t_{0}$ and $t_{1}$ is computed from the pressure. For the system with hybrid pressure levels, it is sufficient to compute the average surface pressure $\left(\overline{p_{s}}\right)_{i}$ for each cell $i$ :

$$
\left(\overline{p_{s}}\right)_{i}=\iint_{A_{i}} p_{s}(\lambda, \beta) \cos \beta \mathrm{d} \lambda \mathrm{d} \beta / A_{i} \quad[\mathrm{~Pa}]
$$


Together with the hybrid coefficients, this defines the mass in a box $i$ at level $k$ :

$$
m_{i, k}=\left[\Delta a_{k}+\Delta b_{k}\left(\overline{p_{s}}\right)_{i}\right] A_{i} R^{2} / g \quad[\mathrm{~kg}]
$$

where $R$ is the radius of the earth $(\mathrm{m}), g$ the acceleration of gravity $\left(\mathrm{m} / \mathrm{s}^{2}\right)$, and $A_{i}$ the area of the box defined in (3). If the meteorological model provides a spectral field for the natural logarithm of the surface pressure, it should be evaluated on a fine mesh, followed by transformation into surface pressure and numerical quadrature. Optionally, the mass distributions could be slightly adjusted with a multiplication factor to impose a global mass conservation.

\subsection{Computation of the vertical fluxes}

In this stage the vertical mass fluxes are computed for the time interval $\left[t_{0}, t_{1}\right]$. We assume that the temporal resolution of the available spectral data is such that for the center of the time interval the flux could be computed. The computed flux will be used for the entire time interval, without using temporal interpolations.

The vertical flux $\dot{\eta} \partial p / \partial \eta$ is computed by integrating the continuity equation (4) from the top of the model to the desired $\eta$ :

$$
\dot{\eta} \frac{\partial p}{\partial \eta}=-\frac{\partial p}{\partial t}-\int_{0}^{\eta} \nabla \cdot\left(v \frac{\partial p}{\partial \eta}\right) \mathrm{d} \eta \quad[\mathrm{Pa} / \mathrm{s}]
$$

In the hybrid coordinate system, one can use that the surface pressure tendency is computed from (8) with $\eta=1$ and boundary condition of zero flux through the surface, so that the flux through the bottom $\eta_{k+1 / 2}$ of a grid box $i$ at level $k$ is equal to:

$$
\begin{aligned}
& \Phi_{w}\left(A_{i}, \eta_{k+1 / 2}\right)=\frac{R^{2}}{g} \iint_{A_{i}}\left(\dot{\eta} \frac{\partial p}{\partial \eta}\right)_{k+1 / 2} \cos \beta \mathrm{d} \beta \mathrm{d} \lambda \\
& =\frac{R^{2}}{g} \iint_{A_{i}}\left[\left(\sum_{j=1}^{n_{\text {lev }}} \Omega_{j}\right) b_{k+1 / 2}-\sum_{j=1}^{k} \Omega_{j}\right] \cos \beta \mathrm{d} \beta \mathrm{d} \lambda \quad[\mathrm{kg} / \mathrm{s}]
\end{aligned}
$$

where

$$
\Omega_{j}=\boldsymbol{\nabla} \cdot\left(\boldsymbol{v}_{j} \Delta p_{j}\right)=D_{j}\left(\Delta a_{j}+\Delta b_{j} p_{s}\right)+\frac{\boldsymbol{V}_{j}}{\cos \beta} \cdot\left(\boldsymbol{\nabla}\left(\ln p_{s}\right)\right) p_{s} \Delta b_{j}
$$

In spectral models it is common that the velocity vector $\boldsymbol{V}=\boldsymbol{v} \cos \beta$ is available in spectral form, otherwise it can be derived from the divergence and vorticity. The singularity in $\boldsymbol{V} / \cos \beta$ at the pole is thus circumvented. The asymptotic value of $v$ at the poles can be set to an average of surrounding values. The gradient $\nabla\left(\ln p_{s}\right)$ of the natural logarithm of the surface pressure is hard to obtain from an interpolated pressure field, but easily derived from spherical harmonics. 


\subsection{Computation of the horizontal fluxes}

Similar to the vertical fluxes, the horizontal fluxes are computed from meteorological fields valid at a time centered within $\left[t_{0}, t_{1}\right]$, and used over the whole time interval. The flux through a vertically oriented surface $S$ is equal to:

$$
\Phi_{h}=\frac{1}{g} \iint_{S} \boldsymbol{n} \cdot \boldsymbol{v} \frac{\partial p}{\partial \eta} \mathrm{d} \eta \mathrm{d} l \quad[\mathrm{~kg} / \mathrm{s}]
$$

with $\boldsymbol{v}$ the horizontal velocity vector, $\boldsymbol{n}$ the normal vector on the surface, and $l$ the horizontal coordinate $(\mathrm{m})$. In the hybrid vertical coordinate system, the fluxes through a longitudinally or latitudinally oriented surface between two half levels are given by:

$$
\begin{array}{lll}
\Phi_{u}\left(\Delta \beta_{i}, k\right) & =\frac{R}{g} \int_{\Delta \beta_{i}} \frac{U(\lambda, \beta, k)}{\cos \beta}\left[\Delta a_{k}+p_{s}(\lambda, \beta) \Delta b_{k}\right] \mathrm{d} \beta & {[\mathrm{kg} / \mathrm{s}]} \\
\Phi_{v}\left(\Delta \lambda_{i}, k\right) & =\frac{R}{g} \int_{\Delta \lambda_{i}} V(\lambda, \beta, k)\left[\Delta a_{k}+p_{s}(\lambda, \beta) \Delta b_{k}\right] \mathrm{d} \lambda & {[\mathrm{kg} / \mathrm{s}]}
\end{array}
$$

where $U$ and $V$ are the components of $\boldsymbol{V}=\boldsymbol{v} \cos \beta$. The integrals can be approximated by numerical quadrature after evaluation of $\boldsymbol{V}$ on a fine grid. At the poles, the limiting values of $U / \cos \beta$ along the meridians need to be evaluated for computation of $\Phi_{u}$; the zero length of $\Delta \lambda_{i}$ ensures vanishing of $\Phi_{v}$ here.

\subsection{Conservation of mass}

For each grid box in the tracer model, the mass change between the start and end of the time interval should equal the net flux through the six boundaries:

$$
m\left(t_{1}\right)-m\left(t_{0}\right)=\left[\delta_{\lambda} \Phi_{u}+\delta_{\beta} \Phi_{v}+\delta_{\eta} \Phi_{w}\right] \Delta t
$$

where the $\delta_{\star}$ denote the difference operators between opposite boundaries of a grid box. For two reasons, the balance will not be matched exactly by the variables computed in 4.1-4.3. First, the fluxes through the boundaries are computed from meteorological fields valid at a discrete time rather than over the complete interval. Secondly, both the mass distributions and the fluxes are subject to interpolation errors. To produce a consistent set of mass distributions and fluxes, some of these variables therefore need to be adjusted.

Taking all variables in (15) into account, the largest absolute errors occur in the net horizontal fluxes. This is because the horizontal velocities are much stronger than the vertical velocities, and they hardly vary from one box to its neighbors. The net horizontal flux is therefore computed from differences between large and almost equal numbers. The adjustment procedure proposed here is to add small corrections to the horizontal fluxes only, leaving the mass distributions and vertical fluxes unchanged. The procedure consists of adding a correction flux 
$\boldsymbol{F}=\left(F_{u}, F_{v}\right)$ to the horizontal fluxes $\boldsymbol{\Phi}_{h}=\left(\Phi_{u}, \Phi_{v}\right)$ such that for all grid boxes the following expression holds:

$$
\boldsymbol{\delta}_{h} \cdot \boldsymbol{F}=\left[m\left(t_{1}\right)-m\left(t_{0}\right)\right] / \Delta t-\boldsymbol{\delta}_{h} \cdot \boldsymbol{\Phi}_{h}-\delta_{\eta} \Phi_{w}
$$

where $\delta_{h}=\left(\delta_{\lambda}, \delta_{\beta}\right)$ is the horizontal difference operator. This correction can be applied to every model layer independent of other layers. The system of equations (16) is under-determined, since the number of correction fluxes is about twice the number of grid boxes. Therefore, the correction flux can be obtained from (16) using a least squares solver, able to handle sparse matrices. If the grid is regular (same number of grid cells in each latitudinal or longitudinal band), another procedure could be applied by requiring that the correction flux is divergence free, completely defined by a potential $\Psi$ according to $\boldsymbol{F}=\boldsymbol{\delta}_{h} \Psi$. With this assumption, system (16) is transformed in a discrete Poisson equation, easily solved with a discrete 2D Fourier transform. The result is a correction field $\boldsymbol{F}$, periodically continued at the boundaries. For a global model, the boundary condition of periodically fluxes in longitudinal direction is valid as such. In the latitudinal direction, the fluxes over the poles should remain zero (periodically with a fixed value), which can be simply achieved by subtraction of the polar flux from each longitudinal column of the grid. Since the corrections are in general small in comparison with the horizontal fluxes (less than a percent), the new horizontal fluxes hardly differ from the original fluxes.

The procedure described here provides balanced mass fluxes for a global tracer model. If mass fluxes are to be derived for a limited area only, the procedure has to be slightly changed. The sub domain is supposed to be a high resolution or zooming area of a global, coarse grid model, for which mass distribution and fluxes are available. Ideally, each cell in the sub domain is covered by one cell of the coarse grid only; in an extreme situation, the sub domain is covered by a single cell of the coarse grid. Mass distribution and fluxes for the high resolution grid are computed following the equations in 4.1-4.3. The first step in balancing the mass is now to bring the computed values in agreement with the values of the coarse grid. That is, if a box of the coarse grid covers $n$ boxes in the high resolution grid, the total mass in the $n$ small boxes should equal the mass in the large box, and similarly for fluxes across the boundaries. A simple scale factor is sufficient here, since the difference between values in the global grid and the sum over high resolution cells is probably small (if not, the global variables are inaccurate and should be recalculated with more accurate quadrature). The second step is to compute the corrections to the horizontal fluxes in the same way as described before, but now for each each cell of the coarse grid, with corrections forced to zero at the boundaries.

\section{Model results}

The impact of computing mass fluxes directly from spectral fields instead of already interpolated gridded fields has been examined with the TM3 model. The model input for TM3 is derived from ECMWF meteorological data at a 
temporal resolution of 6 hours and using 31 hybrid $\sigma /$ pressure levels between the surface and $10 \mathrm{hPa}$. The horizontal grid of TM3 is regular with a resolution of $2.5^{\circ} \times 2.5^{\circ}$. Mass distributions and mass fluxes are computed for each grid cell, although in the advection routine (second moment scheme [1]) some grid cells around the poles are actually joined to avoid Courant numbers exceeding 1.

The horizontal mass fluxes for TM3 were in the past computed from wind fields interpolated to a grid of $1^{\circ} \times 1^{\circ}$ degrees. A small difference between the net mass flux into a column of grid cells and the actual mass change observed from the surface pressure tendency could not be avoided. Therefore, a correction field was added to the horizontal fluxes, distributed over the layers with ratios following the absolute fluxes. Vertical fluxes were only derived afterwards given the corrected horizontal fluxes and the mass tendency in each layer. In the new system, the fluxes are computed from spectral fields (T106), and where necessary interpolated to a grid of $0.25^{\circ} \times 0.25^{\circ}$ for numerical quadrature. Figure 1 shows an example of the error in vertical fluxes derived from the (uncorrected) horizontal fluxes and mass tendency. The derived vertical fluxes are compared with the vertical fluxes computed using the method described in section 4.2. The error is reduced significantly in almost all layers of the model when using spectral fields instead of interpolated fields. Especially at the top of the model, where the vertical fluxes are small in comparison with the horizontal flow and therefore not so easily derived from horizontal convergence only, the error is reduced by $50 \%$. The errors increase smoothly towards the bottom of the model, and are comparable near the surface where all wind components approach zero. The error in the vertical flux is removed up to machine precision by the mass balance correction described in 4.4 .

The difference between mass fluxes computed from interpolated and spectral fields in tracer simulations has been re-examined for experiments described in detail in $[2,7]$. In that study, ozone observations from commercial aircraft flights in the mid latitude lower-most stratosphere were compared with simulations with TM3 and other models. It was concluded that the model performance significantly depends on the preprocessing of the fluxes, and that interpolations should be avoided. Figure 2 shows an example of the improvement of the performance if the fluxes are computed from spectral fields. This figure shows the mean plus and minus the standard deviation for the measured and simulated ozone concentrations. The simulations are either based on interpolated winds, with optionally a correction using vertical velocities [2], or on spectral data. The simulated variability of ozone improves significantly in comparison with the simulation based on interpolated fields, especially when the spectral data is used. While in the case of interpolated fields the range of ozone values was almost twice as broad as actually measured, the new simulations roughly cover the same variability range as observed. 


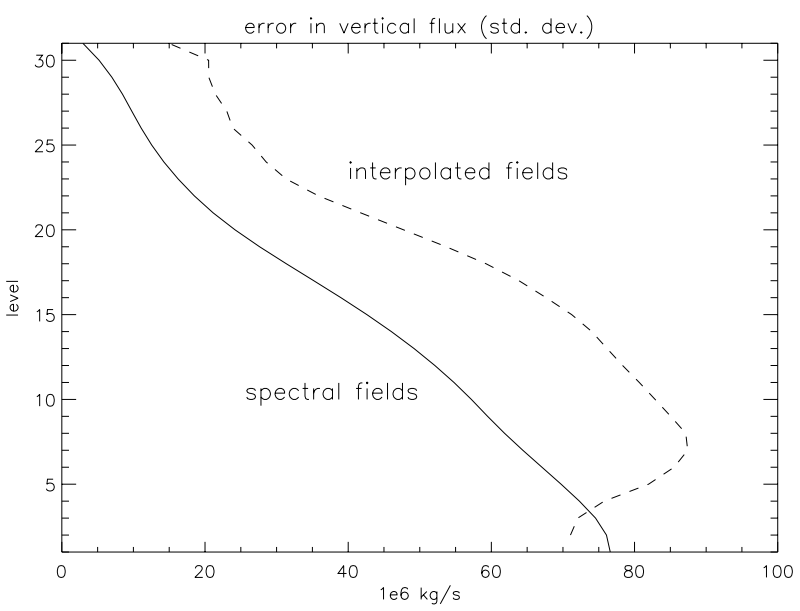

Fig. 1. Error in vertical flux computed from uncorrected horizontal convergence and mass change, if compared with vertical flux computed from spectral fields (section 4.2). Computed for may 26, 1996 over four 6-hour intervals.

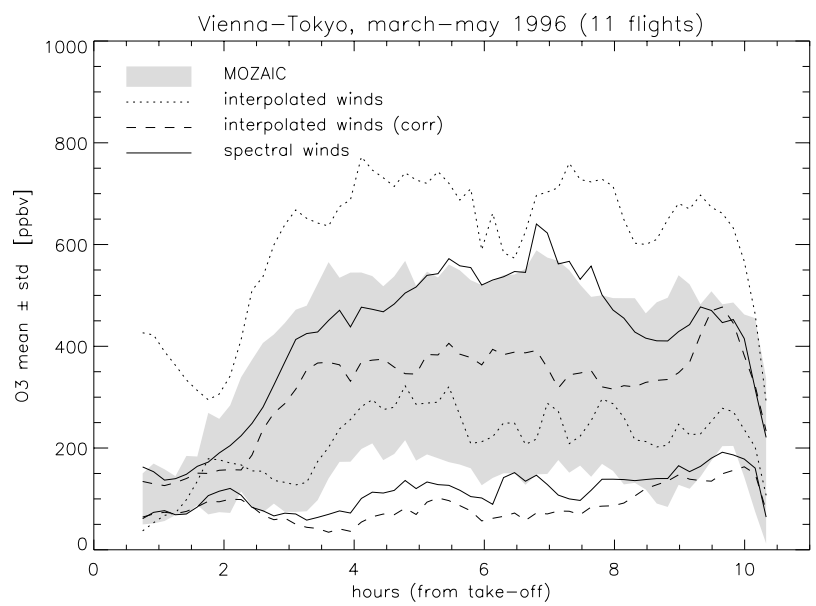

Fig. 2. Example of the impact of the new method for computation of the fluxes on an ozone simulation. Mean plus and minus standard deviation of ozone for flights ViennaTokyo during March-May 1996: observations, TM3 with interpolated horizontal winds, corrected with vertical velocities (see [2]), and using spectral fields. 


\section{Conclusions}

A method for the computation of mass fluxes for a Eulerian box model from spectral fields of vorticity, divergence, and surface pressure has been described. The procedure is applicable for global as well as a limited area Eulerian models with arbitrary horizontal resolution. The fluxes are computed using numerical quadrature over spectral fields evaluated on a fine grid, since direct integration of spectral functions or products of spectral functions is not feasible analytically.

The computed fluxes satisfy the condition of mass conservation over a finite time interval, whose length is determined by the time resolution of the driving meteorological model or the archiving frequency. The availability of spectral fields has been explored to compute vertical fluxes directly, without the need for using interpolated horizontal wind fields. Small adjustments need to be made to the horizontal fluxes to get conservation of mass. Without such small adjustments, the mass is not conserved exactly due to numerical inaccuracies, and the need for fluxes to be valid over a time interval rather than at a discrete time.

Ozone simulations with the TM3 model showed significant improvement in the fluxes when derived from spectral data instead of interpolated wind fields. The error in the horizontal mass convergence is reduced by $20-50 \%$ in almost all layers of the model. Comparison of TM3 simulations with ozone data observed during aircraft flights showed good agreement of modeled and measured variability in concentrations.

\section{References}

1. Prather, M.: Numerical advection by conservation of second-order moments. J. Geophys. Res. 91 (1986) 6671-6681

2. Bregman, A., Krol, M., Teyssèdre, H., Norton, W., Iwi, A., Chipperfield, M., Pitari, G., Sundet, J., Lelieveld, J.: Chemistry-transport model comparision with ozone observations in the midlatitude lowermost stratosphere. J. of Geophys. Res. 106 (2001) 17,479

3. Berntsen, T.K., Isaksen, I.S.: A global three-dimensional chemical transport model for the troposphere: 1. model description and $\mathrm{CO}$ and ozone results. J. Geophys. Res. 102 (1997) 21,239-21,280

4. Holton, J.R.: An introduction to dynamic meteorology. third edn. Volume 48 of International Geophysics Series. Academic Press (1992)

5. Stockwell, D.Z., Chipperfield, M.P.: A tropospheric chemical-transport model: Development and validation of the model transport schemes. Q.J.R. Meteorol. Soc. 125 (1999) 1747-1783

6. Simmons, A.J., Burridge, D.M.: An energy and angular-momentum conserving vertical finite-difference scheme and hybride vertical coordinates. Mon. Weath. Rev. 109 (1981) 758-766

7. Bregman, A., Krol, M., Segers, A., van Velthoven, P.: A new mass flux processing method for global models and the effect on ozone distributions in the upper troposphere and lower stratosphere. Submitted to GRL (2002) 